surgical procedure with minimal hemodynamic impact, ensuring a reliable block with quick onset and low risk of local anesthetic toxicity. In the case allowed adequate hemodynamic management to avoid mitral regurgitation deterioration and optimize graft perfusion. Continuous spinal anesthesia was suitable for the surgical procedure and contributed to an individualized management essential to the outcome of the patient.

\section{AGAINST ALL ODDS: EPIDURAL HAEMATOMA IN A MINOR HEPATIC METASTASECTOMY}

S Pereira*, H Gouveia, S Nunes, M Luís. Hospital Dr. Nélio Mendonça, Funchal, Portugal

\subsection{6/rapm-2021-ESRA.58}

Background and Aims Epidural analgesia (EA) in patients undergoing liver resection remains controversial because of the increased risk of epidural haematoma (EH), although extremely rare [1]. We report a case of $\mathrm{EH}$ in a patient submitted to a hepatic metastesectomy, after EA.

Methods A 71-year-old, diagnosed with metastatic cholangiocarcinoma and previously submitted to cephalic pancreatoduodenectomy, was admitted for elective metastasectomy $(2 \times 3$ centimeters). Her history included hypertension on bisoprolol and chemotherapy two years previously. Preoperative assessment revealed minor changes, as described in table 1 . The patient consented a combination of EA with general anaesthesia and a lower thoracic epidural catheter (T9-T10) was uneventfully placed.

Results A two-hour surgery was performed successfully, with insignificant blood loss. On postoperative day 2, the patient underwent emergent reintevertion due to severe hemoperitoneum but no vascular bleeding point was detected and therefore, coagulopathy was assumed. After transfusion of 3 units of fresh frozen plasma (FFP) and 1 platelet concentrate (PC), haemorrhage resolved and the epidural catheter was removed the next day, considering a 12 hour interval since the last administration of prophylactic enoxaparin. 18 hours later, the patient presented with severe lumbar and inferior limb pain and paraparesis. A CT scan revealed an

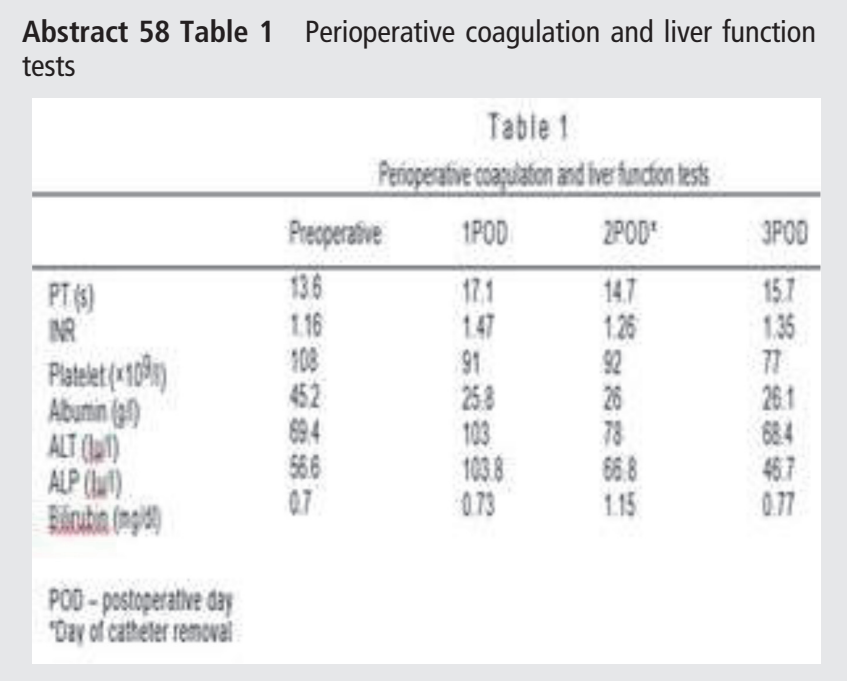

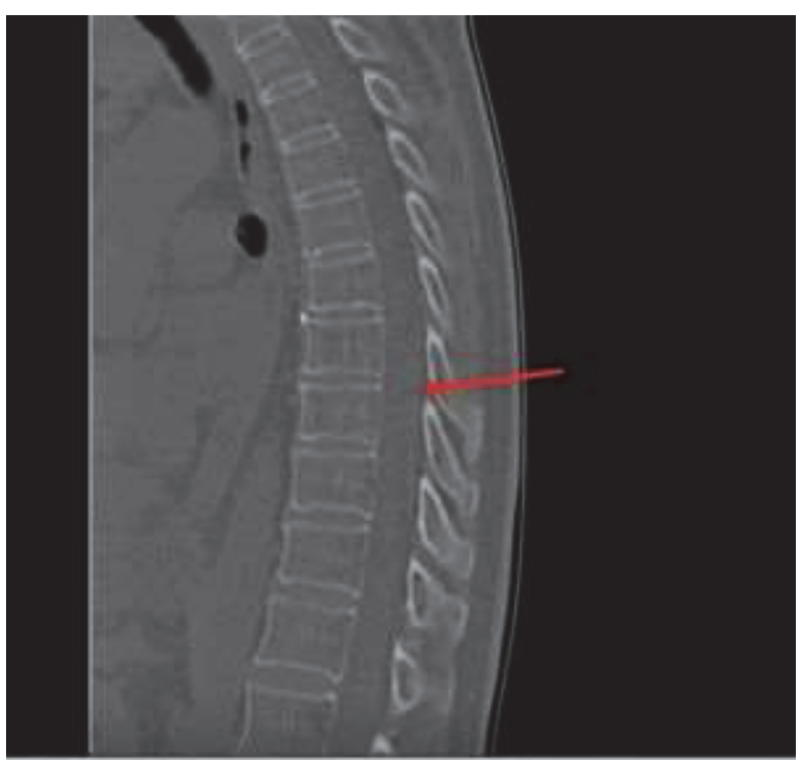

Abstract 58 Figure 1 Thoracic and lumbar CT scan. A hyperintense lesion displacing the cord anteriorly at T8-T10
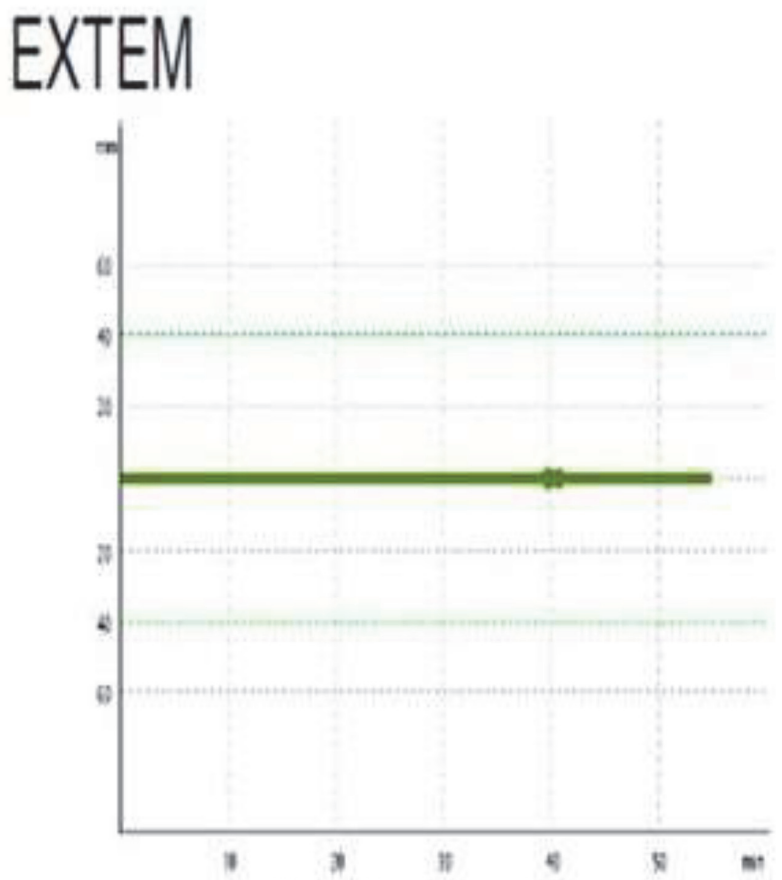

Abstract 58 Figure 2 ROTEM ${ }^{8}$ Thromboelastometry showing no activation of dot formation in EXTEM

EH from T8-T10, and emergent laminectomy was performed. 1 PC, 4 FFP and 1000U of prothrombin complex concentrate were administered, ROTEM guided. After several months of rehabilitation, the patient managed to walk unassisted.

Conclusions Despite following every recommendation for epidural catheter handling, masked coagulopathy can prevail and thus a high level of EH suspicion should always subsist. 